\title{
Logistics management provides greater efficiency, governance and compliance
}

\author{
Francesco Ferrara ${ }^{1}$ (D . Priscilla Santilli ${ }^{1}$ Antonio Vitiello ${ }^{1} \cdot$ Giampiero Forte $^{2} \cdot$ Vilma D'Aiuto $^{1}$
}

Received: 4 February 2021 / Accepted: 12 May 2021 / Published online: 13 June 2021

() The Author(s), under exclusive licence to Springer Nature Switzerland AG 2021

\begin{abstract}
During the 2020 Congress of the Italian Society of Hospital Pharmacy (Sifo 2020), there was a heated debate about whether it is more convenient to dispense healthcare directly in hospitals and public facilities, or indirectly, through the use of external private pharmacies. The former solution is called "direct dispensing" (DD), while the latter is called "dispensing on commission" (DPC). The strengths and advantages of DD over DPC are many: greater therapeutic appropriateness due to direct control of treatment plans, cost savings from external commissions, and greater clinical sharing of treatment by all healthcare personnel. The main weakness is the organization of a complex warehouse. As the number of chronic patients and advanced therapies increases, direct care requires a new structure and logistics system to manage large administrative, accounting, and information flows. The purpose of this article is to compare the two logistic models implemented in Italy in order to structure a computerized model that can increase the efficiency of the National Health System (NHS).
\end{abstract}

Keywords Assessment $\cdot$ Clinical $\cdot$ Direct distribution $\cdot$ Governance $\cdot$ Health System $\cdot$ Logistic $\cdot$ Pharmacy $\cdot$ Sustainability . Technology

\begin{abstract}
Abbreviations
DD Direct distribution

DPC Dispensing on commission

SIFO Congress of the Italian Society of Hospital Pharmacy

NHS National Health System

NRP Nominative Request Procedure

DSS Social and Sanitary District - This is a public territorial unit of the Local Health Authority located close to the patient's residence
\end{abstract}

\section{Impacts on practice}

The logistics process ensures cost-effectiveness and high therapeutic appropriateness.

Francesco Ferrara

francesco.ferrara@uslumbria1.it

1 Pharmaceutical department, Usl Umbria 1, A.Migliorati street, 06132 Perugia, Italy

2 Pharmaceutical department, Asl Roma 5, Colleferro, Rome, Italy
Delivery of therapies to the patient's home leads to increased compliance.

Delivery of therapies to the patient's home leads to increased compliance.

Greater appropriateness means less waste and more savings.

The computerised process allows absolute precision in terms of traceability.

\section{Introduction}

The proper organization of healthcare technology from provider to patient is an essential step in improving the allocation of available resources, while also increasing the quality of healthcare. In this direction, logistics and technological innovation play a fundamental role in supporting the clinic. For example, logistics can ensure greater therapeutic adherence to treatment by the patient. This is because the patient can pick up the medicine at his or her place of residence and have greater compliance without losing working hours and travelling long distances. In addition, in highly disabling diseases such as chronic inflammatory diseases, the distribution of health technology at home can improve the quality of life of patients and families. The complexity of treatments 
today requires flexible models tailored to patient needs. In the creation of a new and efficient health care system, the continuous collaboration between pharmacists and clinical physicians is essential: the central and unequivocal goal is to ensure the best possible patient care while ensuring the sustainability of choices [1].

This manuscript describes a new drug distribution modality that can be adopted by all countries with a public health system. The objective is to highlight how direct distribution of drugs (DD) by the National Health System (NHS), compared with a "dispensing on commission" (DPC), can lead to economic advantages and therapeutic appropriateness.

\section{The new computerised health logistics model}

The congress of the Italian society of hospital pharmacists Sifo 2020 highlighted the possibility of how a logistics service can lead to significant savings. Directly managed drugs can be an attractive solution not only in terms of savings. In fact, the control of clinical therapeutic appropriateness, in a constantly evolving pharmaceutical world, requires that the pharmacist verifies therapies and incorporates his skills and responsibilities with those of the prescribing physician [2].

The model adopted by ASL Roma 5 provides for the centralization of all district pharmacies in a single territorial pharmacy. This is done through a contract with an external provider who provides furniture, non-healthcare personnel, and vehicles for the implementation of this project, which brings the medication directly to the patient's home. If the model proposed at ASL Roma 5 is fully convincing in theory, in practice it leaves many questions. The main problem, is that the external supplier is integrated into the healthcare company without defining roles and responsibilities. This model, moreover, does not define the necessary personnel and the relative cost; the possible difficulties in managing large territorial areas; the modalities of the data storage system; the levels of control before arriving at the dispensation. These are aspects of fundamental importance to plan for a new organization of territorial pharmaceutical assistance.

The model proposed from USL Umbria 1 exceeds these problems thanks to the computerized management shared from all the sanitary staff. The adopted model in fact is realized through a contract with an external supplier that is taken care of all the handling of the warehouse and the relative administrative registrations. The only point of interaction is through the management program: the pharmacist once validated the prescription activates a logistic process for which only the external supplier is responsible. We therefore have the solution to the previous problems: costs and personnel are borne by the external supplier and are part of the supply contract; the logistics management program keeps track of all warehouse movements and economic flows; everything is traced and controlled by a NHS pharmacist. Deliveries are made directly to the health district near the patient's home. This also avoids non-deliveries due to the absence of the patient and additional control by additional healthcare personnel downstream in the logistics process.

The patient is thus placed at the center of the care system. The computer system allows the physician to enter the new patient's data, attach the care plan, and share the information with the pharmacist and the patient's health district.

The only cost is the one related to the supply contract: there are no additional personnel costs, on the contrary, there are considerable savings from other professional figures no longer needed, such as those of administrative and warehouse workers. The process is more streamlined and fluid without loss of data.

External outsourcing provides dedicated and experienced staff to support healthcare companies. No costs are incurred other than those of the contract. In this way the NHS pharmacist does not have to worry about warehousing and administration issues, but he can do their job of assessing treatments and sharing any treatment problems with prescribers. It will be the job of the warehouse staff not only to stock products but also to prepare the supply of patients for dispatch to the various health districts with scheduled deliveries (Fig. 1). It is evident that, thanks to the computer system, there is no dispersion and/or loss of data.

\section{The nominative request procedure (NRP)}

There are three main stages:

(1) The clinician draws up the electronic request with the document base attached (prescription, therapy plan, etc.);

(2) The pharmacist checks and verifies the prescription, the patient's personal data and previous therapies, the stock of products in the warehouse;

(3) The external supplier executes the validated request, prepares the product, organises the shipment and records all the handling data.

Instant traceability with administrative unloading is therefore simultaneous with product delivery. In practice, this new management avoids all the criticalities of a postdispatch registration: e.g. stock mismatching and possible data loss. The new procedure therefore interfaces perfectly between warehouse management and the generated reporting flow (Fig. 2).

In order to have further control and avoid errors, the external supplier also employs its own pharmacist who further checks the request and supports the NHS pharmacist if needed. 
Fig. 1 The pharmaceutical logistics process, four steps: in the first two the external supplier checks, stores the products in the warehouse and uploads them to the warehouse management system. Then, after the NHS pharmacist informally validates the requests, the external supplier schedules the shipment according to the delivery area and in the last step makes all administrative records

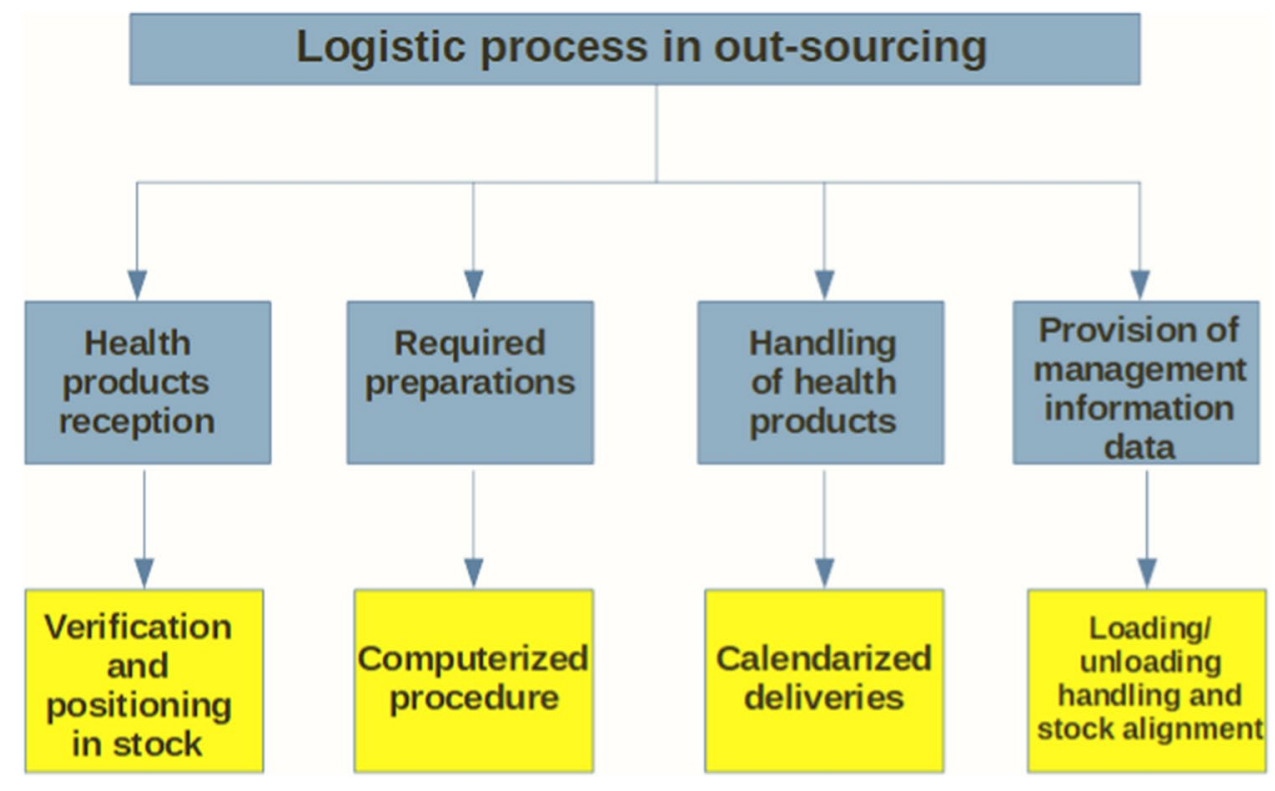

Fig. 2 Flowchart about the RNP computerized request procedur: The DSS enters the patient's prescription into the NRP system; the NHS pharmacist validates the prescription (consults with other healthcare professionals if necessary); the external supplier prepares and schedules the shipment to the DSS

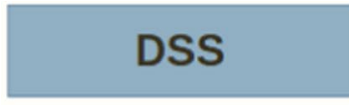

\section{NHL Pharmacist}

Outsourced Service Manager

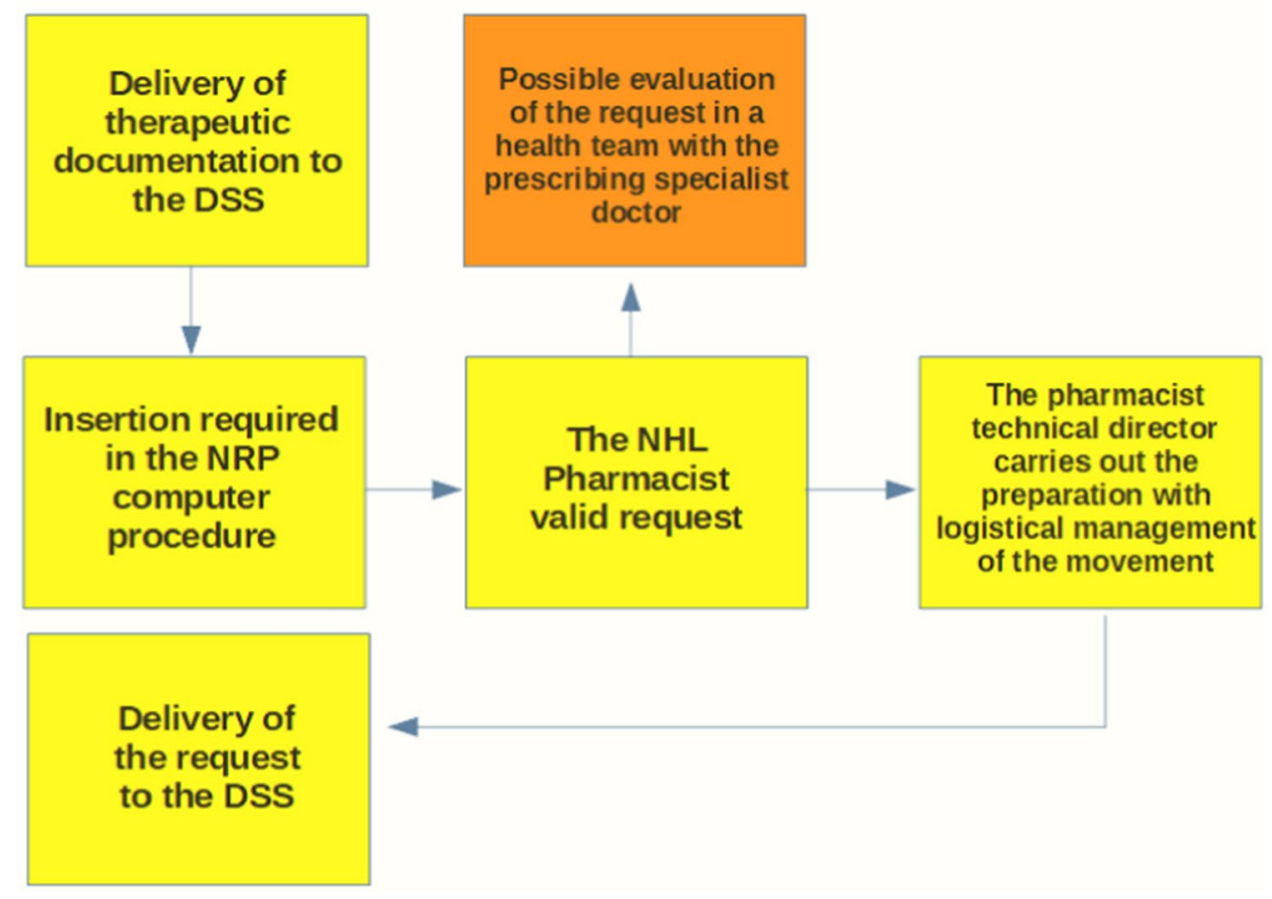

The electronic procedure also includes the management of returns. The process is similar but reversed: the health district enters a return request, it is checked by the NHS pharmacist, the external supplier schedules the take-back and updates the warehouse management system.

\section{Comparison of the two logistics models}

The NRP computerised procedure allows a better management than the one proposed to the Asl Roma 5. Moreover, the mediation of the DSS, which enters the request in a 
management program, guarantees the perfect efficiency of this drug dispensing system. The support of the management program allows the correct registration of the request with certain times, the correct unloading and dispatch. These operations are therefore automated, eliminating possible operator errors. The pharmacist will be more focused on evaluating the forms and validating the request for further processing, which is the responsibility of the external supplier. It is intuitive that in this way there is no waste and everything is tracked automatically without a subsequent download with inevitable data loss.

\section{Logistic warehouse and pandemic period}

In addition, in 2020, the logistical organization of the warehouse facilitated the remote distribution of medicines, without contagion or risk due to the recent ongoing Sars-CoV-2 pandemic. Patients did not have to make long trips and travel outside their district of residence. Contacts were all telematic and the electronic information flow facilitated processes without increasing inconvenience to patients. At the same time, delivery of supplies to hospitals was also facilitated, especially with regard to the anti-COVID-19 emergency drugs used on an experimental basis (colchicine, tocilizumab, pirfenidone, sacubitril, chloroquine, azithromycin) [3-18].

Controlling stocks ensured that many of these drugs, used in other chronic therapies, were not missing from patients' usual treatments. In fact, many healthcare companies have had to face the problem of shortages of anti-rheumatic and antiretroviral drugs, leaving patients untreated. All this is strictly avoided thanks to the perfect knowledge and management of stocks in a computerised way [19, 20].

\section{Conclusions}

In the current healthcare environment of reduced financial resources, healthcare expenditure management is of paramount importance. The reorganization of the distribution processes of healthcare goods from the supplier to the patient's home is an essential factor to improve not only the allocation of available resources but also patient care and therapeutic adherence. The issues of logistics and technological and managerial innovation in hospital and territorial pharmaceutical services are acquiring a central role in supporting the clinic and in pursuing the objectives of increasing the efficiency and quality of health services.

The work performed at USL Umbria 1, with a computerised management system, certainly allows greater centralisation and capture of prescriptions. Besides, the collaboration of the DSS allows an additional check before the delivery of the therapy to the patient, as well as ensuring the proper functioning of the computerized system.

The only cost is the contract for the external warehouse. The quantification of the savings generated depends on the amount of the contract that is signed. In a later publication, it will be possible to discuss all direct, indirect and intangible costs resulting from this new methodology. However, we can certainly say that this new computerised methodology could lead to considerable cost savings, an increase in prescriptive appropriateness and a reduction in registration errors, with greater traceability of the entire process and greater compliance, and therefore therapeutic adherence, for patients undergoing chronic treatment.

Acknowledgements We would like to thank the Pharmacy Direction of Usl Umbria 1 and Asl Roma 5 for their collaboration in the development of this important report.

Funding No funding has been received for its preparation.

Conflicts of interest None of the Authors have conflicts of interest to disclose.

\section{References}

1. Italian Ministry of Health. Guidelines for preparing and transmitting files to NHIS 2017. http://www.salute.gov.it/imgs/C 17_pubblicazioni_1653_allegato.pdf [Accedssed 10 April 2021].

2. Ciacca A. Malattie Croniche: riorganizzazione dei percorsi di cura e dell'assistenza farmaceutica territoriale. Boll SIFO 2020;66(6):346-354.

3. Ferrara F, Santilli P, Vitiello A, D'Aiuto V. From HTA to HTCLA: The Clinic and Logistics to Support Health Technology Assessments. Hosp Pharm. 2020. doi:https://doi.org/10.1177/00185 78720978733.

4. Ferrara F, Santilli P, Bartolini L, Vitiello A, Pennacchia A, Di Croce $S$, et al. L'importanza del magazzino logistico in periodo di pandemia: l'esperienza dell'USL Umbria 1 durante covid-19. Recenti Prog Med. 2021;112(3):219-24.

5. Vitiello A, Ferrara F, Pelliccia C, Granata G, La Porta R. Cytokine storm and colchicine potential role in fighting SARS-CoV-2 pneumonia? Ital J Med. 2020;14(2):88-94.

6. Ferrara F, Granata G, Pelliccia C, La Porta R, Vitiello A. The added value of pirfenidone to fight inflammation and fibrotic state induced by SARS-CoV-2: Anti-inflammatory and anti-fibrotic therapy could solve the lung complications of the infection? Eur J Clin Pharmacol. 2020;1-4.

7. Vitiello A, La Porta R, Ferrara F. Sacubitril, valsartan and SARSCoV-2. BMJ Evid Based Med. 2020 Jul;27:bmjebm-2020.

8. Ferrara F, Porta R, Santilli P, D'Aiuto V, Vitiello A. Are multiple sclerosis therapies safe in severe acute respiratory syndrome coronavirus 2 times? Indian J Pharmacol. 2020;52(5):441-2.

9. Vitiello A, Pelliccia C, Ferrara F. Drugs acting on the reninangiotensin system and SARS-CoV-2. Drug Discov Today. 2021;:S1359-6446(21)00037 - 4.

10. Vitiello A, La Porta R, Ferrara F. Scientific hypothesis and rational pharmacological for the use of sacubitril/valsartan in cardiac damage caused by COVID-19. Med Hypotheses. 2021;147:110486. 
11. Ferrara F, Vitiello A. Efficacy of synthetic glucocorticoids in COVID-19 endothelites. Naunyn Schmiedebergs Arch Pharmacol. 2021;1-5.

12. Vitiello A, Ferrara F. Remdesivir versus ritonavir/lopinavir in COVID-19 patients. Ir J Med Sci. 2020;1-2.

13. Vitiello A, Ferrara F, Porta R. Remdesivir. and COVID-19 infection, therapeutic benefits or unnecessary risks? Ir J Med Sci. 2021;1-2.

14. Ferrara F, Porta R, D'Aiuto V, Vitiello A. Remdesivir and COVID19. Ir J Med Sci. 2020;1-2.

15. Vitiello A, La Porta R, D’Aiuto V, Ferrara F. Pharmacological approach for the reduction of inflammatory and prothrombotic hyperactive state in COVID-19 positive patients by acting on complement cascade. Hum Immunol. 2021;S0198-8859(21)00014 - 8 .

16. Ferrara F, Vitiello A. Potential pharmacological approach in the regulation of ACE-2 and DPP-IV in diabetic COVID-19 patient. Ital J Med. 2020;15:1.
17. Vitiello A, Ferrara F. Pharmacological agents to therapeutic treatment of cardiac injury caused by Covid-19. Life Sci. 2020;262:118510.

18. Vitiello A, Ferrara F. Therapeutic Strategies for SARS-CoV-2 acting on ACE-2. Eur J Pharm Sci. 2021;156:105579.

19. Vitiello A, La Porta R, Ferrara F. Correlation between the use of statins and COVID-19: what do we know? BMJ Evid Based Med. 2020; bmjebm-2020-111589.

20. Ferrara F. Antirheumatic in SARS-CoV-2: benefit or risk? Ital J Med. 2020;14(2):114-5.

Publisher's Note Springer Nature remains neutral with regard to jurisdictional claims in published maps and institutional affiliations. 